\title{
EXTENSIONS OF EXTENDED SYMMETRIC RINGS
}

\author{
TaI KeUn KwaK
}

\begin{abstract}
An endomorphism $\alpha$ of a ring $R$ is called right (left) symmet$r i c$ if whenever $a b c=0$ for $a, b, c \in R, a c \alpha(b)=0(\alpha(b) a c=0)$. A ring $R$ is called right (left) $\alpha$-symmetric if there exists a right (left) symmetric endomorphism $\alpha$ of $R$. The notion of an $\alpha$-symmetric ring is a generalization of $\alpha$-rigid rings as well as an extension of symmetric rings. We study characterizations of $\alpha$-symmetric rings and their related properties including extensions. The relationship between $\alpha$-symmetric rings and (extended) Armendariz rings is also investigated, consequently several known results relating to $\alpha$-rigid and symmetric rings can be obtained as corollaries of our results.
\end{abstract}

\section{Introduction}

Recall that a ring is reduced if it has no nonzero nilpotent elements. Lambek called a ring $R$ symmetric [13] provided $a b c=0$ implies $a c b=0$ for $a, b, c \in R$. Every reduced ring is symmetric ([16, Lemma 1.1]) but the converse does not hold by [2, Example II.5]. Cohn called a ring $R$ rever sible [5] if $a b=0$ implies $b a=0$ for $a, b \in R$. Historically, some of the earliest known results about reversible rings (although not so called at the time) were due to Habeb [6]. It is obvious that commutative rings are symmetric and symmetric rings are reversible; but the converses do not hold by [2, Examples I.5 and II.5] and [14, Examples 5 and 7].

Another generalization of a reduced ring is an Armendariz ring. Rege and Chhawchharia called a ring $R$ Armendariz [15] if whenever any polynomials $f(x)=a_{0}+a_{1} x+\cdots+a_{m} x^{m}, g(x)=b_{0}+b_{1} x+\cdots+b_{n} x^{n} \in R[x]$ satisfy $f(x) g(x)=0$, then $a_{i} b_{j}=0$ for each $i$ and $j$. This nomenclature was used by them since it was Armendariz who initially showed that a reduced ring always satisfies this condition ([3, Lemma 1]).

For a ring $R$ with a ring endomorphism $\alpha: R \rightarrow R$, a skew polynomial ring (also called an Ore extension of endomorphism type) $R[x ; \alpha]$ of $R$ is the ring obtained by giving the polynomial ring over $R$ with the new multiplication $x r=\alpha(r) x$ for all $r \in R$.

Received March 6, 2007.

2000 Mathematics Subject Classification. Primary 16W20, 16U80; Secondary 16S36.

Key words and phrases. reduced rings, symmetric rings, (extended) Armendariz rings.

This work was supported by the Daejin University Research Grants in 2007. 
The Armendariz property of a ring was extended to skew polynomial rings but with skewed scalar multiplication in [8,9]: For an endomorphism $\alpha$ of a ring $R, R$ is called $\alpha$-Armendariz (resp. $\alpha$-skew Armendariz) if for $p=\sum_{i=0}^{m} a_{i} x^{i}$ and $q=\sum_{j=0}^{n} b_{j} x^{j}$ in $R[x ; \alpha], p q=0$ implies $a_{i} b_{j}=0$ (resp. $\left.a_{i} \alpha^{i}\left(b_{j}\right)=0\right)$ for all $0 \leq i \leq m$ and $0 \leq j \leq n$.

On the other hand, an endomorphism $\alpha$ of a $\operatorname{ring} R$ is called rigid [12] if $a \alpha(a)=0$ implies $a=0$ for $a \in R$, and $R$ is an $\alpha$-rigid ring [7] if there exists a rigid endomorphism $\alpha$ of $R$. Note that any rigid endomorphism of a ring is a monomorphism, and $\alpha$-rigid rings are reduced rings by [7, Proposition 5]. Any $\alpha$-rigid ring is $\alpha$-Armendariz [7, Proposition 6], but the converse is not true, in general; every $\alpha$-Armendariz ring is $\alpha$-skew Armendariz, but the converse does not hold by [9, Theorem 1.7 and Example 1.8]. In [8, Proposition 3], $R$ is an $\alpha$-rigid ring if and only if $R[x ; \alpha]$ is reduced.

Motivated by the above, in this paper we introduce the notion of an $\alpha$ symmetric ring for an endomorphism $\alpha$ of a ring $R$, as a generalization of $\alpha$ rigid rings and an extension of symmetric rings, and study $\alpha$-symmetric rings and their related properties. The relationship between $\alpha$-symmetric rings and extended Armendariz rings is also investigated. Consequently, several known results are obtained as corollaries of our results.

Throughout this paper $R$ denotes an associative ring with identity and $\alpha$ denotes a nonzero and non identity endomorphism, unless specified otherwise.

\section{Properties of $\alpha$-symmetric rings}

We begin with the following definition.

Definition 2.1. An endomorphism $\alpha$ of a ring $R$ is called right (left) symmetric if whenever $a b c=0$ for $a, b, c \in R, a c \alpha(b)=0(\alpha(b) a c=0)$. A ring $R$ is called right (left) $\alpha$-symmetric if there exists a right (left) symmetric endomorphism $\alpha$ of $R$. $R$ is $\alpha$-symmetric if it is both right and left $\alpha$-symmetric.

Observe that every subring $S$ with $\alpha(S) \subseteq S$ of a right $\alpha$-symmetric ring is also right $\alpha$-symmetric; and any domain $R$ is $\alpha$-symmetric for any endomorphism $\alpha$ of $R$, but the converse does not hold (see Example 2.7(1) below).

The next example shows that the concept of $\alpha$-symmetric is not left-right symmetric.

Example 2.2. Let $\mathbb{Z}$ be the ring of integers. Consider a ring

$$
R=\left\{\left(\begin{array}{cc}
a & b \\
0 & c
\end{array}\right) \mid a, b, c \in \mathbb{Z}\right\} .
$$

Note that for $A=\left(\begin{array}{ll}0 & 1 \\ 0 & 1\end{array}\right)$ and $B=\left(\begin{array}{ll}1 & 1 \\ 0 & 0\end{array}\right) \in R$, we have $A B=O$ but $B A \neq O$. Thus $R$ is not reversible, and so $R$ is not symmetric.

(i) Let $\alpha: R \longrightarrow R$ be an endomorphism defined by 


$$
\alpha\left(\left(\begin{array}{ll}
a & b \\
0 & c
\end{array}\right)\right)=\left(\begin{array}{ll}
a & 0 \\
0 & 0
\end{array}\right) .
$$

If $A B C=O$ for $A=\left(\begin{array}{ll}a & b \\ 0 & c\end{array}\right), B=\left(\begin{array}{rr}a^{\prime} & b^{\prime} \\ 0 & c^{\prime}\end{array}\right)$ and $C=\left(\begin{array}{rr}a^{\prime \prime} & b^{\prime \prime} \\ 0 & c^{\prime \prime}\end{array}\right) \in R$, then we get $a a^{\prime} a^{\prime \prime}=0$ and so $a a^{\prime \prime} a^{\prime}=0$. Thus this yields $A C \alpha(B)=O$, and hence $R$ is right $\alpha$-symmetric. However, for $A=\left(\begin{array}{ll}0 & 1 \\ 0 & 1\end{array}\right), B=\left(\begin{array}{ll}1 & 1 \\ 0 & 0\end{array}\right)$ and $C=\left(\begin{array}{ll}1 & 0 \\ 0 & 1\end{array}\right) \in R$ with $A B C=O$, we have $\alpha(B) A C \neq O$, and thus $R$ is not left $\alpha$-symmetric.

(ii) Let $\beta: R \longrightarrow R$ be an endomorphism defined by

$$
\beta\left(\left(\begin{array}{ll}
a & b \\
0 & c
\end{array}\right)\right)=\left(\begin{array}{ll}
0 & 0 \\
0 & c
\end{array}\right) .
$$

By the similar method to (i), we can show that $R$ is left $\beta$-symmetric. However, for $A=\left(\begin{array}{ll}1 & 0 \\ 0 & 1\end{array}\right), B=\left(\begin{array}{ll}0 & 1 \\ 0 & 1\end{array}\right)$ and $C=\left(\begin{array}{ll}1 & 1 \\ 0 & 0\end{array}\right) \in R$ with $A B C=O$, we have $A C \beta(B) \neq O$, and thus $R$ is not right $\beta$-symmetric.

Proposition 2.3. (1) For a ring $R, R$ is right $\alpha$-symmetric if and only if $A B C=0$ implies $A C \alpha(B)=0$ for any three nonempty subsets $A, B$ and $C$ of $R$.

(2) Let $R$ be a reversible ring. $R$ is right $\alpha$-symmetric if and only if $R$ is left $\alpha$-symmetric.

Proof. (1) It suffices to show that $A B C=0$ for any three nonempty subsets $A, B$ and $C$ of $R$ implies $A C \alpha(B)=0$, when $R$ is right $\alpha$-symmetric. Let $A B C=0$. Then $a b c=0$ for $a \in A, b \in B$ and $c \in C$, and hence $a c \alpha(b)=0$ by the condition. Thus $A C \alpha(B)=\sum_{a \in A, b \in B, c \in C} a c \alpha(b)=0$.

(2) Let $a b c=0$ for $a, b, c \in R$. If $R$ is right $\alpha$-symmetric, then $a c \alpha(b)=0$. Since $R$ is reversible, we have $\alpha(b) a c=0$ and hence $R$ is left $\alpha$-symmetric. The converse is similar.

Example 2.2 shows that the condition " $R$ is reversible" in Proposition 2.3(2) cannot be dropped as well as there exists a right symmetric endomorphism $\alpha$ of a ring $R$ such that $R$ is not symmetric. The next example provides that there exists a commutative reduced ring $R$ which is not $\alpha$-symmetric for some endomorphism $\alpha$ of $R$.

Example 2.4. Let $\mathbb{Z}_{2}$ be the ring of integers modulo 2 and consider a ring $R=$ $\mathbb{Z}_{2} \oplus \mathbb{Z}_{2}$ with the usual addition and multiplication. Then $R$ is a commutative reduced ring, and so $R$ is symmetric. Now, let $\alpha: R \rightarrow R$ be defined by $\alpha((a, b))=(b, a)$. Then $\alpha$ is an automorphism of $R$. For $a=(1,0), b=$ $(0,1), c=(1,1) \in R, a b c=0$ but $a c \alpha(b)=(1,0) \neq 0$, and thus $R$ is not right $\alpha$-symmetric. 
Recently, the reversible property of a ring is extended to a ring endomorphism in [4] as follows: An endomorphism $\alpha$ of a ring $R$ is called right reversible if whenever $a b=0$ for $a, b \in R, b \alpha(a)=0$. A ring $R$ is called right $\alpha$-reversible if there exists a right reversible endomorphism $\alpha$ of $R$. The notion of an $\alpha$ reversible ring is a generalization of $\alpha$-rigid rings as well as an extension of reversible rings.

Theorem 2.5. Let $R$ be a right $\alpha$-symmetric ring. Then we have the following.

(1) For $a, b, c \in R, a b c=0$ implies $a c \alpha^{n}(b)=0, b c \alpha^{n}(a)=0$, and $a b \alpha^{n}(c)=$ 0 for any positive integer $n$, especially, $R$ is a right $\alpha$-reversible ring.

(2) Let $\alpha$ be a monomorphism of $R$. Then we have the following.

(i) $R$ is a symmetric ring.

(ii) For $a, b, c \in R$ abc $=0$ implies $\alpha^{n}(a) b c=0$ and $a \alpha^{n}(b) c=0$ for any positive integer $n$. Conversely, if $\alpha^{m}(a) b c=0, a \alpha^{m}(b) c=0$, or $a b \alpha^{m}(c)=0$ for some positive integer $m$, then $a b c=0$.

Proof. (1) Let $a, b, c \in R$ with $a b c=0$. Since $R$ is right $\alpha$-symmetric, $a c \alpha(b)=$ 0 . Then $0=a c \alpha(b)=(a c) \alpha(b) \cdot 1$ implies $a c \alpha^{2}(b)=0$. Continuing this process, we have $a c \alpha^{n}(b)=0$ for any positive integer $n$. Similarly, $1 \cdot a(b c)=0$ implies $b c \alpha(a)=0$. By the same method as above, we obtain $b c \alpha^{n}(a)=0$ for any positive integer $n$. Finally, $0=a b c=(a b) c \cdot 1$ implies $a b \alpha(c)=0$, and thus $a b \alpha^{n}(c)=0$ for any positive integer $n$.

(2) Suppose that $\alpha$ is a monomorphism. (i): Let $a, b, c \in R$ with $a b c=0$. Then $a c \alpha(b)=0$, and so $\alpha(b) \alpha(a c)=0$ by (1). Since $\alpha$ is a monomorphism, $b a c=0$ and $a c b=0$. Thus $R$ is symmetric. (ii): Note that $R$ is symmetric and so reversible. Let $a b c=0$. Then $b c \alpha^{n}(a)=0$ by (1). Since $R$ is reversible, $\alpha^{n}(a) b c=0$. Next, from $a b c=0$ we have $a c \alpha^{n}(b)=0$ by (1). Since $R$ is symmetric, $a \alpha^{n}(b) c=0$. Conversely, if $\alpha^{m}(a) b c=0$ for some positive integer $m$ then $\alpha^{m}(a) \alpha^{m}(b c)=\alpha^{m}(a b c)=0$ by (i), and thus $a b c=0$, since $\alpha$ is a monomorphism. Similarly, if $a \alpha^{m}(b) c=0$ then $a c \alpha^{m}(b)=0$, since $R$ is symmetric. Hence $\alpha^{m}(a c) \alpha^{m}(b)=0$ by (i), and $a c b=0$ and so $a b c=0$. By the same method as above, we can obtain that $a b \alpha^{m}(c)=0$ implies $a b c=0$.

Corollary 2.6. Every symmetric ring is reversible.

Notice that for any positive integer $n$, " $a \alpha^{n}(b)=0$ " is equivalent to " $a R \alpha^{n}(b)$ $=0$ ", when $R$ is a right $\alpha$-symmetric ring with $a b=0$ for $a, b \in R$ : For, $a b r=0$ implies $\operatorname{ar} \alpha(b)=0$ for any $r \in R$. This shows that $\operatorname{arc}^{n}(b)=0$ for any positive integer $n$ and any $r \in R$ from Theorem 2.5(1), and thus $a R \alpha^{n}(b)=0$.

We remark that the converse of Theorem 2.5(1) does not hold. For example, the ring $R$ with an endomorphism $\alpha$ in Example 2.2(1) is right $\alpha$-symmetric. However, for $A=\left(\begin{array}{ll}0 & 1 \\ 0 & 1\end{array}\right)=B \in R$, we have $A \alpha^{n}(B)=O=B \alpha^{n}(A)$ for any positive integer $n$ but $A B \neq O$. 
In the next example, part (1) shows that there exists a right $\alpha$-symmetric ring $R$ for an automorphism $\alpha$, but $R$ is not semiprime and so not $\alpha$-rigid, and part (2) illuminates that there exists a commutative domain and an $\alpha$ symmetric ring $R$, but $R$ is not $\alpha$-rigid where $\alpha$ is not a monomorphism.

Example 2.7. (1) Consider a ring

$$
R=\left\{\left(\begin{array}{cc}
a & b \\
0 & a
\end{array}\right) \mid a, b \in \mathbb{Z}\right\} .
$$

Let $\alpha: R \rightarrow R$ be an endomorphism defined by

$$
\alpha\left(\left(\begin{array}{ll}
a & b \\
0 & a
\end{array}\right)\right)=\left(\begin{array}{rr}
a & -b \\
0 & a
\end{array}\right) .
$$

Clearly, $R$ is not semiprime and hence $R$ is not $\alpha$-rigid. Note that $\alpha$ is an automorphism. Moreover, $R$ is right $\alpha$-symmetric: Indeed, let $A B C=O$ for $A=\left(\begin{array}{cc}a & b \\ 0 & a\end{array}\right), B=\left(\begin{array}{cc}a^{\prime} & b^{\prime} \\ 0 & a^{\prime}\end{array}\right)$ and $C=\left(\begin{array}{cc}a^{\prime \prime} & b^{\prime \prime} \\ 0 & a^{\prime \prime}\end{array}\right) \in R$, then we get $a a^{\prime} a^{\prime \prime}=0$ and $a a^{\prime} b^{\prime \prime}+a b^{\prime} a^{\prime \prime}+b a^{\prime} a^{\prime \prime}=0$. If $a=0$ then $b a^{\prime} a^{\prime \prime}=0$, if $a^{\prime}=0$ then $a b^{\prime} a^{\prime \prime}=0$, and $a^{\prime \prime}=0$ then $a a^{\prime} b^{\prime \prime}=0$. These imply that $a a^{\prime \prime} a^{\prime}=0$ and $-a a^{\prime \prime} b^{\prime}+a b^{\prime \prime} a^{\prime}+b a^{\prime \prime} a^{\prime}=0$. Thus $A C \alpha(B)=O$, and hence $R$ is right $\alpha$-symmetric.

(2) Let $R=F[x]$ be the polynomial ring over a field $F$. Define $\alpha: R \rightarrow R$ by $\alpha(f(x))=f(0)$ where $f(x) \in R$. Then $R$ is a commutative domain (and so reduced), but $\alpha$ is not a monomorphism. Since $R$ is a domain, $R$ is right $\alpha$-symmetric for any endomorphism $\alpha$ of $R$. However, $R$ is not $\alpha$-rigid by [8, Example 5(2)].

The class of semiprime rings and the class of right $\alpha$-symmetric rings do not depend on each other by Example 2.4 and Example 2.7(1). There exists a skew polynomial ring $R[x ; \alpha]$ over a symmetric ring $R$ which is not a symmetric ring. For example, consider the commutative ring $R=\mathbb{Z}_{2} \oplus \mathbb{Z}_{2}$ and the automorphism $\alpha$ of $R$ defined by $\alpha((a, b))=(b, a)$, as in Example 2.4. Then $R$ is a symmetric ring, but $R[x ; \alpha]$ is not reversible hence not symmetric: Indeed, for $p=(1,0), q=(0,1) x \in R[x ; \alpha]$, we get $p q=0$ but $0 \neq(0,1) x=q p$.

However, we have the following theorem.

Theorem 2.8. (1) For a ring $R, R$ is $\alpha$-rigid if and only if $R$ is semiprime and right $\alpha$-symmetric and $\alpha$ is a monomorphism.

(2) If the skew polynomial ring $R[x ; \alpha]$ of a ring $R$ is a symmetric ring, then $R$ is $\alpha$-symmetric.

Proof. (1) Let $R$ be $\alpha$-rigid. Note that any $\alpha$-rigid ring is reduced and $\alpha$ is a monomorphism by [7, p. 218]. We show that $R$ is right $\alpha$-symmetric. Assume that $a b c=0$ for $a, b, c \in R$. Then we obtain $b a c=0$, since $R$ is reduced (and so symmetric). Thus $a c \alpha(b) \alpha(a c \alpha(b))=a c \alpha(b a c) \alpha^{2}(b)=0$. Since $R$ is $\alpha$-rigid, $a c \alpha(b)=0$ and thus $R$ is right $\alpha$-symmetric.

The converse follows from [4, Proposition 2.5(3)] and Theorem 2.5(1). 
(2) Suppose that $a b c=0$ for $a, b, c \in R$. Let $p=a, q=b$ and $h=c x$ in $R[x ; \alpha]$. Then $p q h=a b c x=0 \in R[x ; \alpha]$. Since $R[x ; \alpha]$ is symmetric, we get $0=p h q=(a c) x b=a c \alpha(b) x$, and so $a c \alpha(b)=0$. Thus $R$ is right $\alpha$-symmetric and therefore $R$ is $\alpha$-symmetric by Proposition 2.3(2).

Corollary 2.9 ([10, Proposition 2.7(1)]). A ring $R$ is reduced if and only if $R$ is a semiprime and symmetric ring.

Observe that the class of right $\alpha$-symmetric rings and the class of $\alpha$-Armendariz rings do not depend on each other by Example 2.7(2) and [11, Example $14]$.

Theorem 2.10. Let $R$ be an $\alpha$-Armendariz ring. The following statements are equivalent:

(1) $R[x ; \alpha]$ is symmetric.

(2) $R$ is $\alpha$-symmetric.

(3) $R$ is right $\alpha$-symmetric.

(4) $R$ is symmetric.

Proof. (1) $\Leftrightarrow(4)$ by $[9$, Theorem $3.6(1)]$ and $(1) \Rightarrow(2)$ by Theorem 2.8 (2). $(2) \Rightarrow(3)$ is trivial. Now we show $(3) \Rightarrow(4)$. Suppose $a b c=0$ for $a, b, c \in R$. Then $a c \alpha(b)=0$, and so $a c b=0$ by [9, Proposition $1.3(2)]$. Thus $R$ is symmetric.

The next result is a direct consequence of Theorem 2.10.

Corollary 2.11 ([10, Proposition 3.4]). Let $R$ be an Armendariz ring. $R$ is symmetric if and only if $R[x]$ is symmetric.

Notice that the converse of Theorem 2.8(2) does not hold and the condition " $R$ is an $\alpha$-Armendariz ring" in Theorem 2.10 are not superfluous by Example 2.7(2): Indeed, consider $A=R[y ; \alpha]=F[x][y ; \alpha]$. Now, let $p=1, q=x y$ and $h=x \in A$. Then $p q h=0$, but $p h q=x^{2} y \neq 0$. Hence $A$ is not symmetric. Note that $R$ is not $\alpha$-Armendariz by [9, Example 1.9].

\section{Extensions of $\alpha$-symmetric rings}

Given a ring $R$ and an $(R, R)$-bimodule $M$, the trivial extension of $R$ by $M$ is the ring $T(R, M)=R \oplus M$ with the usual addition and the following multiplication:

$$
\left(r_{1}, m_{1}\right)\left(r_{2}, m_{2}\right)=\left(r_{1} r_{2}, r_{1} m_{2}+m_{1} r_{2}\right) .
$$

This is isomorphic to the ring of all matrices $\left(\begin{array}{cc}r & m \\ 0 & r\end{array}\right)$, where $r \in R$ and $m \in M$ and the usual matrix operations are used.

For an endomorphism $\alpha$ of a ring $R$ and the trivial extension $T(R, R)$ of $R$, $\bar{\alpha}: T(R, R) \longrightarrow T(R, R)$ defined by

$$
\bar{\alpha}\left(\left(\begin{array}{cc}
a & b \\
0 & a
\end{array}\right)\right)=\left(\begin{array}{cc}
\alpha(a) & \alpha(b) \\
0 & \alpha(a)
\end{array}\right)
$$


is an endomorphism of $T(R, R)$. Since $T(R, 0)$ is isomorphic to $R$, we can identify the restriction of $\bar{\alpha}$ on $T(R, 0)$ to $\alpha$.

Note that the trivial extension of a reduced ring is symmetric by [10, Corollary 2.4]. For a right $\alpha$-symmetric ring $R, T(R, R)$ needs not to be an $\bar{\alpha}$ symmetric ring by the next example.

Example 3.1. Consider the right $\alpha$-symmetric ring

$$
R=\left\{\left(\begin{array}{cc}
a & b \\
0 & a
\end{array}\right) \mid a, b \in \mathbb{Z}\right\} .
$$

in Example 2.7(1) where $\alpha$ is defined by

For

$$
\alpha\left(\left(\begin{array}{ll}
a & b \\
0 & a
\end{array}\right)\right)=\left(\begin{array}{rr}
a & -b \\
0 & a
\end{array}\right) .
$$

$$
A=\left(\begin{array}{ll}
\left(\begin{array}{ll}
1 & 0 \\
0 & 1
\end{array}\right) & \left(\begin{array}{ll}
0 & 0 \\
0 & 0
\end{array}\right) \\
\left(\begin{array}{ll}
0 & 0 \\
0 & 0
\end{array}\right) & \left(\begin{array}{ll}
1 & 0 \\
0 & 1
\end{array}\right)
\end{array}\right), B=\left(\begin{array}{ll}
\left(\begin{array}{ll}
0 & 1 \\
0 & 0
\end{array}\right) & \left(\begin{array}{rr}
-1 & 1 \\
0 & -1
\end{array}\right) \\
\left(\begin{array}{ll}
0 & 0 \\
0 & 0
\end{array}\right) & \left(\begin{array}{ll}
0 & 1 \\
0 & 0
\end{array}\right)
\end{array}\right)
$$

and

$$
C=\left(\begin{array}{cc}
\left(\begin{array}{ll}
0 & 1 \\
0 & 0
\end{array}\right) & \left(\begin{array}{ll}
1 & 1 \\
0 & 1
\end{array}\right) \\
\left(\begin{array}{ll}
0 & 0 \\
0 & 0
\end{array}\right) & \left(\begin{array}{ll}
0 & 1 \\
0 & 0
\end{array}\right)
\end{array}\right) \in T(R, R)
$$

$A B C=O$ but $A C \bar{\alpha}(B) \neq O$. Thus $R$ is not $\bar{\alpha}$-symmetric.

Recall that another generalization of a symmetric ring is a semicommutative ring. A ring $R$ is semicommutative if $a b=0$ implies $a R b=0$ for $a, b \in R$. Historically, some of the earliest results known to us about semicommutative rings (although not so called at the time) was due to Shin [16]. He proved that any symmetric ring is semicommutative ([16, Proposition 1.4]) but the converse does not hold ([16, Example 5.4(a)]). Semicommutative rings were also studied under the name zero insertive by Habeb [6].

Proposition 3.2. Let $R$ be a reduced ring. If $R$ is an $\alpha$-symmetric ring, then $T(R, R)$ is an $\bar{\alpha}$-symmetric ring.

Proof. Let $A B C=O$ for

$$
A=\left(\begin{array}{cc}
a & b \\
0 & a
\end{array}\right), B=\left(\begin{array}{cc}
a^{\prime} & b^{\prime} \\
0 & a^{\prime}
\end{array}\right) \text { and } C=\left(\begin{array}{rr}
a^{\prime \prime} & b^{\prime \prime} \\
0 & a^{\prime \prime}
\end{array}\right) \in T(R, R) .
$$

Then we have

(1) $a a^{\prime} a^{\prime \prime}=0$; and

(2) $a a^{\prime} b^{\prime \prime}+a b^{\prime} a^{\prime \prime}+b a^{\prime} a^{\prime \prime}=0$.

In the following, we freely use the fact that $R$ is a reduced ring if and only if for any $a, b \in R, a b^{2}=0$ (or, $a^{2} b=0$ ) implies $a b=0$; and every reduced ring 
is semicommutative. From Eq.(1), we get $a a^{\prime \prime} a^{\prime}=0$ and $a R a^{\prime} R a^{\prime \prime}=0$. From Eq. (2) $\times a^{\prime \prime}$, we have $a a^{\prime} b^{\prime \prime} a^{\prime \prime}+a b^{\prime}\left(a^{\prime \prime}\right)^{2}+b a^{\prime}\left(a^{\prime \prime}\right)^{2}=0$ and so $a b^{\prime} a^{\prime \prime}+b a^{\prime} a^{\prime \prime}=0$. Then $0=a\left(a b^{\prime} a^{\prime \prime}+b a^{\prime} a^{\prime \prime}\right)=a^{2} b^{\prime} a^{\prime \prime}$, and hence $a b^{\prime} a^{\prime \prime}=0$. So Eq.(2) becomes

(3) $a a^{\prime} b^{\prime \prime}+b a^{\prime} a^{\prime \prime}=0$.

If we multiply Eq.(3) on the left side by $a$, then $0=a^{2} a^{\prime} b^{\prime \prime}=a a^{\prime} b^{\prime \prime}$ and so $b a^{\prime} a^{\prime \prime}=0$. Then $a a^{\prime} a^{\prime \prime}=0, a b^{\prime} a^{\prime \prime}=0, a a^{\prime} b^{\prime \prime}=0$ and $b a^{\prime} a^{\prime \prime}=0$, and hence we obtain $a a^{\prime \prime} \alpha\left(a^{\prime}\right)=0, a a^{\prime \prime} \alpha\left(b^{\prime}\right)=0, a b^{\prime \prime} \alpha\left(a^{\prime}\right)=0$ and $b a^{\prime \prime} \alpha\left(a^{\prime}\right)=0$, since $R$ is $\alpha$-symmetric. Thus $A C \bar{\alpha}(B)=O$ and therefore $T(R, R)$ is $\bar{\alpha}$-symmetric.

Corollary 3.3 ([10, Corollary 2.4$])$. Let $R$ be a reduced ring, then $T(R, R)$ is a symmetric ring.

The trivial extension $T(R, R)$ of a ring $R$ is extended to a ring

$$
T_{n}=\left\{\left(\begin{array}{rrrrr}
a & a_{12} & a_{13} & \cdots & a_{1 n} \\
0 & a & a_{23} & \cdots & a_{2 n} \\
0 & 0 & a & \cdots & a_{3 n} \\
\vdots & \vdots & \vdots & \ddots & \vdots \\
0 & 0 & 0 & \cdots & a
\end{array}\right) \mid a, a_{i j} \in R\right\}
$$

for any $n \geq 3$ and an endomorphism $\alpha$ of a ring $R$ is also extended to the endomorphism $\bar{\alpha}: T_{n} \rightarrow T_{n}$ defined by $\bar{\alpha}\left(\left(a_{i j}\right)\right)=\left(\alpha\left(a_{i j}\right)\right)$.

The following example shows that $T_{n}$ cannot be $\bar{\alpha}$-symmetric for any $n \geq 3$, even if $R$ is an $\alpha$-rigid ring.

Example 3.4. Let $\alpha$ be an endomorphism of an $\alpha$-rigid ring $R$. Note that if $R$ is an $\alpha$-rigid ring, then $\alpha(e)=e$ for $e^{2}=e \in R$ by [7, Proposition 5]. In particular $\alpha(1)=1$. First, we show that $T_{3}$ is not $\bar{\alpha}$-symmetric. For

$$
A=\left(\begin{array}{lll}
1 & 0 & 0 \\
0 & 1 & 0 \\
0 & 0 & 1
\end{array}\right), B=\left(\begin{array}{lll}
0 & 0 & 0 \\
0 & 0 & 1 \\
0 & 0 & 0
\end{array}\right), C=\left(\begin{array}{lll}
0 & 1 & 0 \\
0 & 0 & 0 \\
0 & 0 & 0
\end{array}\right) \in T_{3},
$$

$A B C=O$. But we have $A C \bar{\alpha}(B)=C B \neq O \in T_{3}$.

In case of $n \geq 4$, we can also prove that $T_{n}$ is not $\bar{\alpha}$-symmetric by the same method as the above.

Recall that if $\alpha$ is an endomorphism of a ring $R$, then the map $\bar{\alpha}: R[x] \rightarrow$ $R[x]$ defined by $\bar{\alpha}\left(\sum_{i=0}^{m} a_{i} x^{i}\right)=\sum_{i=0}^{m} \alpha\left(a_{i}\right) x^{i}$ is an endomorphism of the polynomial ring $R[x]$ and clearly this map extends $\alpha$. The Laurent polynomial ring $R\left[x, x^{-1}\right]$ with an indeterminate $x$, consists of all formal sums $\sum_{i=k}^{n} a_{i} x^{i}$, where $a_{i} \in R$ and $k, n$ are (possibly negative) integers. The map $\bar{\alpha}: R\left[x, x^{-1}\right] \rightarrow$ $R\left[x, x^{-1}\right]$ defined by $\bar{\alpha}\left(\sum_{i=k}^{n} a_{i} x^{i}\right)=\sum_{i=k}^{n} \alpha\left(a_{i}\right) x^{i}$ extends $\alpha$ and also is an endomorphism of $R\left[x, x^{-1}\right]$. Multiplication is subject to $x r=\alpha(r) x$ and $r x^{-1}=x^{-1} \alpha(r)$.

The following results extend the class of right $\alpha$-symmetric rings.

Theorem 3.5. Let $R$ be a ring.

(1) $R[x]$ is right $\bar{\alpha}$-symmetric if and only if $R\left[x ; x^{-1}\right]$ is right $\bar{\alpha}$-symmetric. 
(2) If $R$ is an Armendariz ring, then $R$ is right $\alpha$-symmetric if and only if $R[x]$ is right $\bar{\alpha}$-symmetric.

Proof. (1) It is sufficient to show necessity. Let $f(x), g(x)$ and $h(x) \in R\left[x ; x^{-1}\right]$ with $f(x) g(x) h(x)=0$. Then there exists a positive integer $n$ such that $f_{1}(x)=$ $f(x) x^{n}, g_{1}(x)=g(x) x^{n}$ and $h_{1}(x)=h(x) x^{n} \in R[x]$, and so $f_{1}(x) g_{1}(x) h_{1}(x)=$ 0 . Since $R[x]$ is right $\bar{\alpha}$-symmetric, we obtain $f_{1}(x) h_{1}(x) \bar{\alpha}\left(g_{1}(x)\right)=0$. Hence $f(x) h(x) \bar{\alpha}(g(x))=x^{-3 n} f_{1}(x) h_{1}(x) \bar{\alpha}\left(g_{1}(x)\right)=0$. Thus $R\left[x ; x^{-1}\right]$ is right $\bar{\alpha}-$ symmetric.

(2) It also suffices to establish necessity. Let $f(x)=\sum_{i=0}^{m} a_{i} x^{i}, g(x)=$ $\sum_{j=0}^{n} b_{j} x^{j}$ and $h(x)=\sum_{k=0}^{l} c_{i} x^{i} \in R[x]$ with $f(x) g(x) h(x)=0$. By [1, Proposition 1], $a_{i} b_{j} c_{k}=0$ for all $i, j$ and $k$, and so $a_{i} c_{k} \alpha\left(b_{j}\right)=0$ since $R$ is Armendariz and right $\alpha$-symmetric. This yields $f(x) h(x) \bar{\alpha}(g(x))=0$, and thus $R[x]$ is right $\bar{\alpha}$-symmetric.

Corollary 3.6. (1) [10, Lemma 3.2(2)] For a ring $R, R[x]$ is symmetric if and only if so is $R\left[x ; x^{-1}\right]$.

(2) [10, Proposition 3.4] Let $R$ be an Armendariz ring. $R$ is symmetric if and only if $R[x]$ is symmetric.

Note that Example 2.2(i) and Example 2.4 show that Armendariz rings and right $\alpha$-symmetric rings do not depend on each other.

For an ideal $I$ of $R$, if $\alpha(I) \subseteq I$ then $\bar{\alpha}: R / I \longrightarrow R / I$ defined by $\bar{\alpha}(a+I)=$ $\alpha(a)+I$ is an endomorphism of a factor ring $R / I$. The homomorphic image of a symmetric ring may not necessarily be symmetric by [10, p.163]. One may conjecture that $R$ is $\alpha$-symmetric if for any right $\alpha$-symmetric nonzero proper ideal $I$ of $R, R / I$ is $\bar{\alpha}$-symmetric, where $I$ is considered as a ring without identity. However the next example erases the possibility.

Example 3.7. For a field $F$, consider a ring $R=\left(\begin{array}{cc}F & F \\ 0 & F\end{array}\right)$ and an endomorphism $\alpha$ of $R$ defined by

$$
\alpha\left(\left(\begin{array}{ll}
a & b \\
0 & c
\end{array}\right)\right)=\left(\begin{array}{rr}
a & -b \\
0 & c
\end{array}\right) .
$$

For a right ideal $I=\left(\begin{array}{cc}0 & F \\ 0 & 0\end{array}\right)$ of $R$, it can be easily checked that $I$ is right $\alpha$-symmetric and the factor ring

$$
R / I=\left\{\left(\begin{array}{cc}
a & 0 \\
0 & c
\end{array}\right)+I \mid a, c \in F\right\}
$$

is reduced. Observe that $R / I$ is $\bar{\alpha}$-symmetric, where $\bar{\alpha}$ is an identity map on $R / I$.

However, for $A=\left(\begin{array}{ll}1 & 0 \\ 0 & 1\end{array}\right), B=\left(\begin{array}{ll}0 & 1 \\ 0 & 0\end{array}\right)$ and $C=\left(\begin{array}{ll}1 & 1 \\ 0 & 0\end{array}\right) \in R$, we have $A C \alpha(B) \neq O$ and $A B C=O$. Thus $R$ is not right $\alpha$-symmetric. 
Theorem 3.8. Let $R$ be a reduced ring and $n$ be any positive integer. If $R$ is right $\alpha$-symmetric with $\alpha(1)=1$, then $R[x] /\left\langle x^{n}\right\rangle$ is a right $\bar{\alpha}$-symmetric ring, where $\left\langle x^{n}\right\rangle$ is the ideal generated by $x^{n}$.

Proof. Let $S=R[x] /\left\langle x^{n}\right\rangle$. If $n=1$, then $S \cong R$. If $n=2$, then $S$ is $\bar{\alpha}-$ symmetric by Proposition 3.2, since $S \cong T(R, R)$. Now, we assume $n \geq 3$. Let $f=a_{0}+a_{1} \bar{x}+\cdots+a_{n-1} \bar{x}^{n-1}, g=b_{0}+b_{1} \bar{x}+\cdots+b_{n-1} \bar{x}^{n-1}$ and $\bar{h}=$ $c_{0}+c_{1} \bar{x}+\cdots+c_{n-1} \bar{x}^{n-1} \in S$ with $f g h=0$, where $\bar{x}=x+\left\langle x^{n}\right\rangle$. Note that $a_{i} b_{j} c_{k} \bar{x}^{i+j+k}=0$ for all $i, j$ and $k$ with $i+j+k \geq n$. Hence it suffices to show the cases $i+j+k \leq n-1$. Since $f g h=0$, we have the following equations:

(1) $a_{0} b_{0} c_{0}=0$.

(2) $a_{0} b_{0} c_{1}+a_{0} b_{1} c_{0}+a_{1} b_{0} c_{0}=0$.

(3) $a_{0} b_{0} c_{2}+a_{0} b_{1} c_{1}+a_{0} b_{2} c_{0}+a_{1} b_{0} c_{1}+a_{1} b_{1} c_{0}+a_{2} b_{0} c_{0}=0$.

$(n-2) a_{0} b_{0} c_{n-2}+a_{0} b_{1} c_{n-3}+\cdots+a_{n-3} b_{1} c_{0}+a_{n-2} b_{0} c_{0}=0$.

$(n-1) a_{0} b_{0} c_{n-1}+a_{0} b_{1} c_{n-2}+\cdots+a_{n-2} b_{0} c_{1}+a_{n-2} b_{1} c_{0}+a_{n-1} b_{0} c_{0}=0$.

Recall that $R$ is a reduced ring if and only if for any $a, b \in R, a b^{2}=0$ implies $a b=0$, and every reduced ring is semicommutative. We use these facts in the following.

Eq. (1) and Eq. $(2) \times b_{0} c_{0}$ give $a_{1}\left(b_{0} c_{0}\right)^{2}=0$, and so $a_{1} b_{0} c_{0}=0$ and $a_{0} b_{0} c_{1}+$ $a_{0} b_{1} c_{0}=0$; multiplying $b_{1} c_{0}$ gives $0=a_{0} b_{1}\left(c_{0}\right)^{2}=a_{0} b_{1} c_{0}$, so we have

$(2)^{\prime} \quad a_{0} b_{0} c_{1}=0, a_{0} b_{1} c_{0}=0$ and $a_{1} b_{0} c_{0}=0$.

From Eqs.(1), (2)' and (3) $\times b_{0} c_{0}$, we get $a_{2} b_{0} c_{0}=0$ and

$(3)^{\prime} a_{0} b_{0} c_{2}+a_{0} b_{1} c_{1}+a_{0} b_{2} c_{0}+a_{1} b_{0} c_{1}+a_{1} b_{1} c_{0}=0$,

in a similar way. If we multiply Eq.(3)' on the right side by $b_{1} c_{0}, b_{0} c_{1}, b_{2} c_{0}$ and $b_{1} c_{1}$ respectively, then we obtain $a_{1} b_{1} c_{0}=0, a_{1} b_{0} c_{1}=0, a_{0} b_{2} c_{0}=0, a_{0} b_{1} c_{1}=0$, and $a_{0} b_{0} c_{2}=0$ in turn.

Inductively we assume that $a_{i} b_{j} c_{k}=0$ for $i+j+k=0,1, \ldots,(n-2)$. We apply the above method to Eq. $(n-1)$. First, the induction hypotheses and Eq. $(n-1) \times b_{0} c_{0}$ give $a_{n-1} b_{0} c_{0}=0$ and

$(n-1)^{\prime} \quad a_{0} b_{0} c_{n-1}+a_{0} b_{1} c_{n-2}+\cdots+a_{n-2} b_{0} c_{1}+a_{n-2} b_{1} c_{0}=0$.

If we multiply Eq. $(n-1)^{\prime}$ on the right side by $b_{1} c_{0}, b_{0} c_{1}, \ldots$, and $b_{1} c_{n-2}$ respectively, then we obtain $a_{n-2} b_{1} c_{0}=0, a_{n-2} b_{0} c_{1}=0, \ldots, a_{0} b_{1} c_{n-2}=0$ and so $a_{0} b_{0} c_{n-1}=0$, in turn. This shows that $a_{i} b_{j} c_{k}=0$ for all $i, j$ and $k$ with $i+j+k=n-1$. Consequently, $a_{i} b_{j} c_{k}=0$ for all $i, j$ and $k$ with $i+j \leq n-1$, and thus $a_{i} c_{k} \alpha^{t}\left(b_{j}\right)=0$ for any positive integer $t$ by Theorem 2.5(1). This yields $f h \bar{\alpha}(g)=0$, and therefore $S$ is right $\bar{\alpha}$-symmetric.

Corollary 3.9 ([10, Theorem 2.3$])$. If $R$ is a reduced ring, then $R[x] /\left\langle x^{n}\right\rangle$ is a symmetric ring for any positive integer $n$.

Let $R$ be an algebra over a commutative ring $S$. Recall that the Dorroh extension of $R$ by $S$ is the ring $D=R \times S$ with operations $\left(r_{1}, s_{1}\right)+\left(r_{2}, s_{2}\right)=$ $\left(r_{1}+r_{2}, s_{1}+s_{2}\right)$ and $\left(r_{1}, s_{1}\right)\left(r_{2}, s_{2}\right)=\left(r_{1} r_{2}+s_{1} r_{2}+s_{2} r_{1}, s_{1} s_{2}\right)$, where $r_{i} \in R$ 
and $s_{i} \in S$. For an endomorphism $\alpha$ of $R$ and the Dorroh extension $D$ of $R$ by $S, \bar{\alpha}: D \longrightarrow D$ defined by $\bar{\alpha}(r, s)=(\alpha(r), s)$ is an $S$-algebra homomorphism.

In the following, we give some other example of right $\alpha$-symmetric rings.

Proposition 3.10. (1) If $e$ is a central idempotent of a ring $R$ with $\alpha(e)=e$ and $\alpha(1-e)=1-e$, then $e R$ and $(1-e) R$ are right $\alpha$-symmetric if and only if $R$ is right $\alpha$-symmetric.

(2) If $R$ is a right $\alpha$-symmetric ring with $\alpha(1)=1$ and $S$ is a domain, then the Dorroh extension $D$ of $R$ by $S$ is $\bar{\alpha}$-symmetric.

Proof. (1) It is enough to show the necessity. Suppose that $e R$ and $(1-e) R$ are right $\alpha$-symmetric. Let $a b c=0$ for $a, b, c \in R$. Then $0=e a b c=a(e b) c$ and $0=(1-e) a b c=a((1-e) b) c$. By hypothesis, we get $0=a c \alpha(e b)=$ $a c e \alpha(a)=e a c \alpha(b)$ and $0=a c \alpha((1-e) b)=a c(1-e) \alpha(b)=(1-e) a c \alpha(b)$. Thus $a c \alpha(b)=e a c \alpha(b)+(1-e) a c \alpha(b)=0$, and therefore $R$ is right $\alpha$-symmetric.

(2) Let $\left(r_{1}, s_{1}\right),\left(r_{2}, s_{2}\right),\left(r_{3}, s_{3}\right) \in D$ with $\left(r_{1}, s_{1}\right)\left(r_{2}, s_{2}\right)\left(r_{3}, s_{3}\right)=0$. Then $r_{1} r_{2} r_{3}+s_{1} r_{2} r_{3}+s_{2} r_{1} r_{3}+s_{3} r_{1} r_{2}+s_{1} s_{2} r_{3}+s_{1} s_{3} r_{2}+s_{2} s_{3} r_{1}=0$ and $s_{1} s_{2} s_{3}=0$. Since $S$ is a domain, we get $s_{1}=0, s_{2}=0$ or $s_{3}=0$. In the following computations, we freely use the assumption that $R$ is right $\alpha$-symmetric with $\alpha(1)=1$. If $s_{1}=0$, then $0=r_{1} r_{2} r_{3}+s_{2} r_{1} r_{3}+s_{3} r_{1} r_{2}+s_{2} s_{3} r_{1}$ and so $0=r_{1}\left(r_{3}+s_{3}\right) \alpha\left(r_{2}+s_{2}\right)=r_{1} r_{3} \alpha\left(r_{2}\right)+r_{1} s_{3} \alpha\left(r_{2}\right)+r_{1} r_{3} s_{2}+r_{1} s_{3} s_{2}$. This yields $\left(r_{1}, s_{1}\right)\left(r_{3}, s_{3}\right) \bar{\alpha}\left(\left(r_{2}, s_{2}\right)\right)=0$. Similarly, let $s_{2}=0$. Then $\left(r_{1}+s_{1}\right) r_{2}\left(r_{3}+s_{3}\right)=$ 0 , and so $\left(r_{1}+s_{1}\right)\left(r_{3}+s_{3}\right) \alpha\left(r_{2}\right)=0$, and hence $r_{1} r_{3} \alpha\left(r_{2}\right)+s_{1} s_{2} \alpha\left(r_{2}\right)+$ $s_{3} r_{1} \alpha\left(r_{2}\right)+s_{1} s_{3} \alpha\left(r_{2}\right)=0$. Thus we have $\left(r_{1}, s_{1}\right)\left(r_{3}, s_{3}\right) \bar{\alpha}\left(\left(r_{2}, s_{2}\right)\right)=0$. Finally, let $s_{3}=0$. Then $\left(r_{1}+s_{1}\right)\left(r_{2}+s_{2}\right) r_{3}=0$, and so $0=\left(r_{1}+s_{1}\right) r_{3}\left(\alpha\left(r_{2}\right)+s_{2}\right)=$ $\left(r_{1} r_{3}+s_{1} s_{3}\right) \alpha\left(r_{2}\right)+s_{2}\left(r_{1} r_{3}+s_{1} r_{3}\right)$. This imply $\left(r_{1}, s_{1}\right)\left(r_{3}, s_{3}\right) \bar{\alpha}\left(\left(r_{2}, s_{2}\right)\right)=0$. Therefore the Dorroh extension $D$ is $\bar{\alpha}$-symmetric.

Corollary 3.11. (1) [10, Proposition 3.6(2)] For an abelian ring $R, R$ is symmetric if and only if $e R$ and $(1-e) R$ are symmetric for every idempotent $e$ of $R$ if and only if $e R$ and $(1-e) R$ are symmetric for some idempotent $e$ of $R$

(2) $[10$, Proposition 4.2(1)] Let $R$ be an algebra over a commutative ring $S$, and $D$ be the Dorroh extension of $R$ by $S$. If $R$ is symmetric and $S$ is a domain, then $D$ is symmetric.

Note that the condition " $\alpha(1)=1$ " in Proposition 3.10(2) cannot be dropped by the next example.

Example 3.12. Let $R=\mathbb{Z}_{2} \oplus \mathbb{Z}_{2}$ and $\alpha: R \longrightarrow R$ be defined by $\alpha((a, b))=$ $(0, b)$. Consider the Dorroh extension $D$ of $R$ by the ring of integers $\mathbb{Z}$. Then we have

$$
((1,0), 0)((1,0),-1)((1,0), 0)=0
$$

in $D$, but

$$
((1,0), 0)((1,0), 0) \bar{\alpha}((1,0),-1)=(-(1,0), 0) \neq 0
$$

in $D$. 
Acknowledgments. The authors would like to thank the referee for helpful comments and suggestions.

\section{References}

[1] D. D. Anderson and V. Camillo, Armendariz rings and Gaussian rings, Comm. Algebra 26 (1998), no. 7, 2265-2272.

[2] $ـ$, Semigroups and rings whose zero products commute, Comm. Algebra 27 (1999), no. $6,2847-2852$.

[3] E. P. Armendariz, A note on extensions of Baer and P.P.-rings, J. Austral. Math. Soc. 18 (1974), 470-473.

[4] M. Başer, C. Y. Hong, and T. K. Kwak, On extended reversible rings, Algebra Colloq. (to appear).

[5] P. M. Cohn, Reversible rings, Bull. London Math. Soc. 31 (1999), no. 6, 641-648.

[6] J. M. Habeb, A note on zero commutative and duo rings, Math. J. Okayama Univ. 32 (1990), 73-76.

[7] C. Y. Hong, N. K. Kim, and T. K. Kwak, Ore extensions of Baer and p.p.-rings, J. Pure Appl. Algebra 151 (2000), no. 3, 215-226.

[8] _ On skew Armendariz rings, Comm. Algebra 31 (2003), no. 1, 103-122.

[9] C. Y. Hong, T. K. Kwak, and S. T. Rizvi, Extensions of generalized Armendariz rings, Algebra Colloq. 13 (2006), no. 2, 253-266.

[10] C. Huh, H. K. Kim, N. K. Kim, and Y. Lee, Basic examples and extensions of symmetric rings, J. Pure Appl. Algebra 202 (2005), no. 1-3, 154-167.

[11] C. Huh, Y. Lee, and A. Smoktunowicz, Armendariz rings and semicommutative rings, Comm. Algebra 30 (2002), no. 2, 751-761.

[12] J. Krempa, Some examples of reduced rings, Algebra Colloq. 3 (1996), no. 4, 289-300.

[13] J. Lambek, On the representation of modules by sheaves of factor modules, Canad. Math. Bull. 14 (1971), 359-368.

[14] G. Marks, Reversible and symmetric rings, J. Pure Appl. Algebra 174 (2002), no. 3, 311-318.

[15] M. B. Rege and S. Chhawchharia, Armendariz rings, Proc. Japan Acad. Ser. A Math. Sci. 73 (1997), no. 1, 14-17.

[16] G. Y. Shin, Prime ideals and sheaf representation of a pseudo symmetric ring, Trans. Amer. Math. Soc. 184 (1973), 43-60.

Department of Mathematics

DAEJIN UNIVERSITY

POCHEON 487-711, KOREA

E-mail address: tkkwak@daej in.ac.kr 